\title{
L-PRF with Allograft in the Treatment of Intrabony Defect
}

Sushma Akula ${ }^{1}$, Vikram Reddy $\mathrm{G}^{2}$, J agadish Reddy ${ }^{3}$, Raja Babu $\mathrm{P}^{4}$

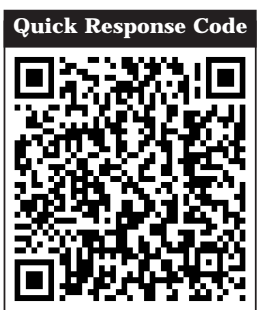

doi: $10.5866 / 2016.8 .10194$

${ }^{1}$ Post Graduate Student,

${ }^{2 \&}{ }^{3}$ Reader

${ }^{4}$ Professor \& HOD

Department of Periodontics,

Kamineni Institute of Dental Sciences,

Narketpally, Nalgonda, Telangana.

\section{Article Info:}

Received: J uly 8, 2016

Review Completed: August 9, 2016

Accepted: September 10, 2016

Available Online: December, 2016 (www.nacd.in)

(c) NAD, 2016 - All rights reserved

E mail for correspondence:

sushma.akula3@gmail.com

\begin{abstract}
:
Background: The most positive outcome of periodontal regenerative procedures in intra bony defect has been achieved with bone grafts. For complete regeneration, delivery of growth factors in a local environment holds a great deal in adjunct to bone grafts. Platelet rich fibrin (PRF) is considered as second generation platelet concentrate, consisting of viable platelets, releasing various growth factors. Leukocyte and platelet- rich fibrin (L-PRF) is a fibrin matrix in which the platelet cytokines, growth factors and cells are trapped and this material has been recruited in reconstruction of various defect. The present case report aims to investigate the effectiveness of autologous PRF along with the use of allograft in the treatment of intra bony defect by comparing clinical and radiographical outcome obtained by combination of PRF and allograft.
\end{abstract}

Key words: Chronic Periodontitis, Periodontal regeneration, Intrabony defects, Platelet rich fibrin, al lograft.

\section{INTRODUCTION:}

Periodontal regeneration is a multifactorial process and requires a multi-dependent sequence of biological events including cell-adhesion, migration, proliferation and differentiation. ${ }^{1}$ The ultimate goal of periodontal therapy is to regenerate the lost periodontal tissues caused by periodontitis. ${ }^{2}$ Various controlled clinical trials have demonstrated that some of the available grafting procedures may result in periodontal regeneration in intrabony defects, but complete and predictable reconstruction of periodontal tissues is still difficult to obtain. ${ }^{3}$ The reason is that periodontium, once damaged has a limited capacity for regeneration. ${ }^{4}$ The most positive outcome of periodontal regeneration procedures in intrabony defect has been achieved with a combination of bone graft and guided tissue regeneration. ${ }^{5,6}$

The complex series of events associated with periodontal regeneration involves recruitment of locally derived progenitor cells subsequently differentiated into periodontal ligament forming cells, cementoblasts or bone forming osteoblasts. Therefore, the key to periodontal regeneration is to stimulate the progenitor cells to reoccupy the defects.

Growth factors are the vital mediators during this process which can induce the migration, 
attachment, proliferation and differentiation of periodontal progenitor cells. Platelet rich fibrin (PRF) may be considered as a second generation platelet concentrate, using simplified protocol, is a recently innovative growth factor delivery medium.

PRF allows to obtain fibrin mesh enriched with platelets and growth factors, from an anticoagulant free blood harvest without any artificial biochemical modification. ${ }^{7}$ The PRF clot forms a strong natural fibrin matrix which concentrates almost all the platelets and growth factors of the blood harvest and shows complex architectures as a healing matrix, including mechanical properties, which no other platel et concentrate can offer. In an article in 2011, Lekovicet al. demonstrated that PRF in combination with bovine porous bone mineral had the ability to increase the regenerative effects in intrabony defects. $^{8}$

In the present case report, we present the clinical and radiographic changes of a patient using L - PRF along with allograft as grafting material in the treatment of periodontal intrabony defect with endodontic involvement.

\section{CASE REPORT}

A 45-year-old systemically healthy female patient reported to the Department of Periodontology, Kamineni Institute of Dental Sciences, Narketpally, Telangana, with a chief complaint of food lodgement in right mandibular back tooth region of the jaw since 1 month. Patient underwent endodontic therapy in relation to \#46 six months back. On periodontal examination, the probing depth on mesiobuccal aspect was $8 \mathrm{~mm}$. The patient also presented with pain on percussion in relation to \#47 and a periapical radiograph was taken which on evaluation, presented with an intrabony defect extending up to middle third of the mesial root of right mandibular first molar (\#46) (Figure 1). The diagnosis was made to be chronic generalized gingivitis with localized periodontitis in relation to \#46

\section{Pre Surgical Therapy:}

Initial therapy consisted of oral hygiene instructions, Scaling and root planning using ultrasonic instruments and curettes were performed. At 4 weeks following phase 1 therapy, a periodontal re-evaluation was performed to confirm the suitability of \#46 tooth for this periodontal surgical procedure.

\section{PRF Preparation:}

PRF preparation requires a centrifuge and collection kit including a 24 gauge needle and $9 \mathrm{ml}$ blood collection tubes. After obtaining the informed consent, whole blood is drawn, into thetubes without anticoagulant and is immediately centrifuged at $3000 \mathrm{rpm}$ for $10 \mathrm{~min}$. The outcome is a fibrin clot containing platelets in the middle of the tube, between the red blood cell layer at the bottom and acellular plasma at the top. This clot is removed from the tube and the attached red blood cells scraped off and discarded. The PRF clot is then placed between two gauzes and pressed thereby squeezing out the fluids in the fibrin clot to obtain a matrix/ membrane which was later mixed with graft material (Figure 2 and 3).

\section{Surgical Procedure:}

An intrasulcular incision was made on buccal and lingual aspect of the tooth of right mandibular teeth (\#45, 46 and 47) (Figure 4). A full thickness mucoperi osteal flap was raised and inner surface of the flap was curetted to remove the granulation tissue. Root surfaces werethoroughly planned using hand instruments. Theright mandibular first molar demonstrated mesial intrabony defect. After removing granulation tissue thoroughly, mesial intrabony defect was found to extend in buccal and apical aspect (Figure 5). The allograft (DFDBA TATA MEMORIAL) with PRF was then condensed (Figure 6). The flap were repositioned to their presurgical levels and sutured with silk utilizing an interrupted technique (Figure 7). After the operation, the patient was prescribed systemic antibiotics (amoxicillin 500 mg tid, 3 days), nonsteroidal anti-inflammatory drug (Hifenac D tid, 3) days) and $0.2 \%$ chlorhexidine rinse (twice a day for 4 weeks). Sutures were removed after 7 days. Clinical healing was normal. The patient was recalled at $1^{\text {st }}$ week, $2^{\text {nd }}$ week, $1^{\text {st }}$ month, $3^{\text {rd }}$ and $6^{\text {th }}$ month. Periapical intraoral radiographs were obtained from the periodontal defect site at baseline, 3 months and 6 months after surgery.

\section{Results:}

In the present case report, the greater reduction in pocket depth was found after 6 months of the follow-up that is from $8 \mathrm{~mm}$ to $2 \mathrm{~mm}$ which is the important clinical outcome for any periodontal regenerative procedures. Radiographs revealed significant bonefill in the intrabony defect compared with measurements at baseline (Figure 8). 


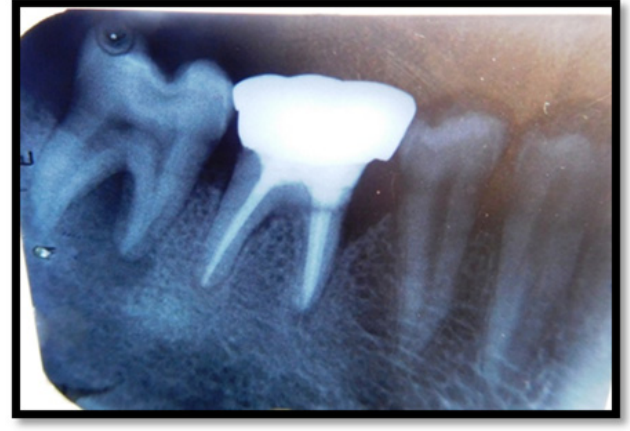

Figure 1: Preoperative radiograph showing an intrabony defect extending up to middle third of the mesial root of right mandibular first molar

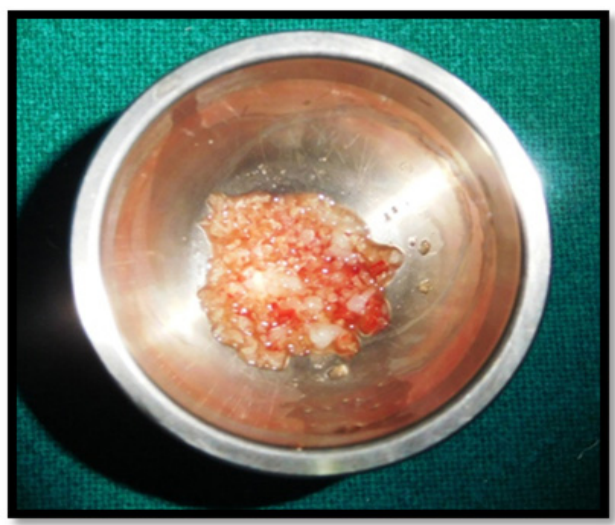

Figure 3: PRF with DFDBA

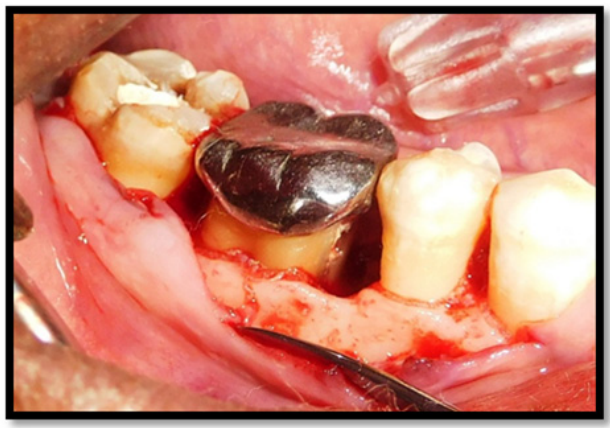

Figure 5: Defect in mesial aspect of \#46

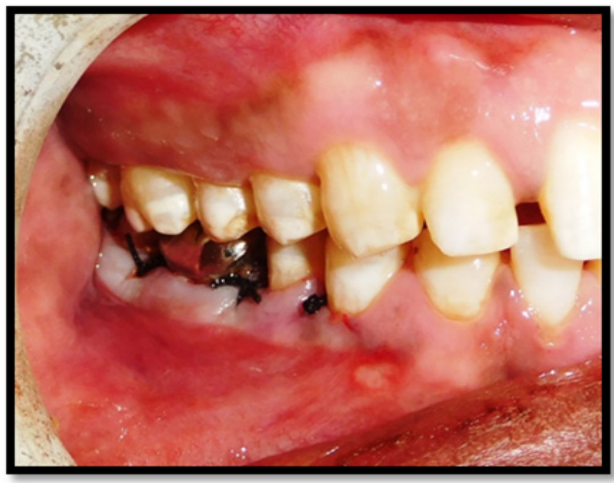

Figure 7: Sutures placed

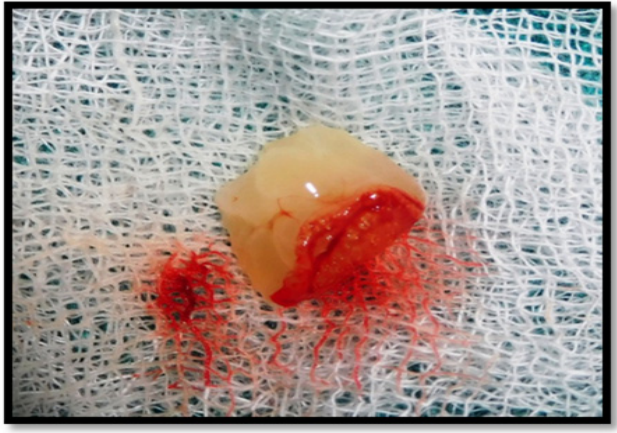

Figure 2: PRF plug

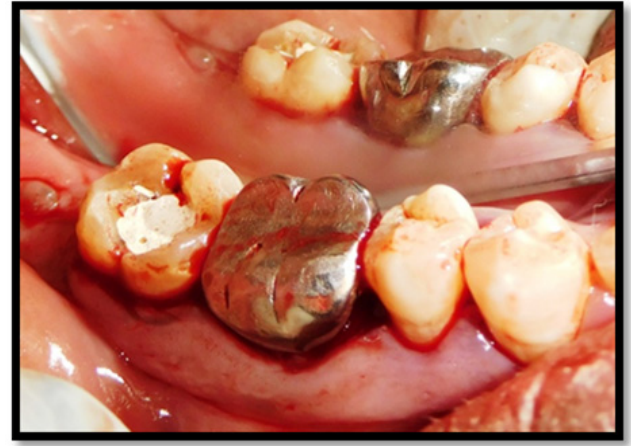

Figure 4: Intra sulcular incisions

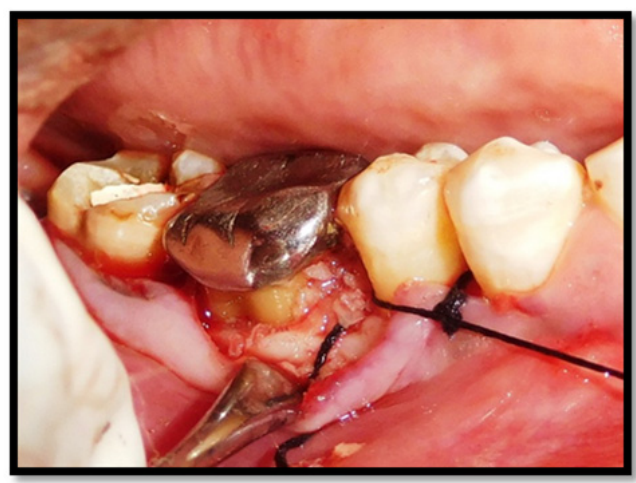

Figure 6: PRF with DFDBA placement

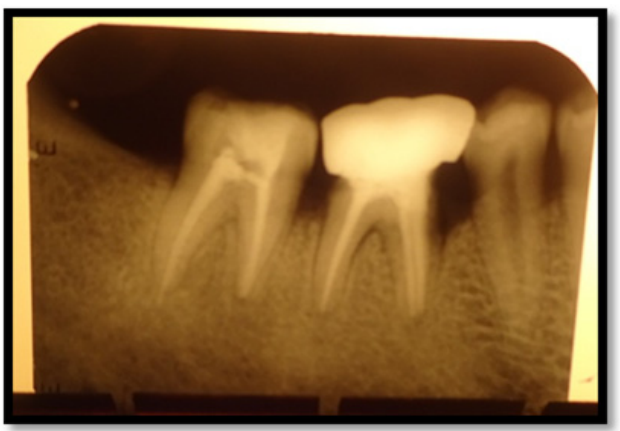

Figure 8: Post operative radiograph showing significant bone fill in the intrabony defect (6 months post operative). 


\section{DISCUSSION:}

The present case report evaluated the clinical efficacy of L-PRF matrix in the treatment of a hard tissue defect. The grafted sites displayed rapid clinical healing, with good tissue contour, color and bone density. In our case, a significant reduction in PPD was found. Significant radiographic bone formation in the periodontal intrabony defect was also observed supporting the role of various growth factors present in the L-PRF matrix in accelerating the soft and hard tissue healing.

Despite of the fact that PRF is a denser and firmer agent than other biological preparations, such as PRP and enamel matrix derivative (EMD), it is still non-rigid to a degree that its space maintaining ability in periodontal defects is non-ideal. When platelets in a concentrated form are added to graft materials, a more predictable outcome is derived. ${ }^{9}$ DFDBA have been used in periodontal therapy for years together. It is known to have an osteogenic potential that is manifested by exposing bone morphogenic protein (BMPs) which presumably have the ability to induce host cells to differentiate into osteoblast. They have been successfully used to reconstruct intraosseous periodontal defects. In another study, PRF in combination in with bone mineral had the ability in increasing the regenerative effects in intrabony defects. ${ }^{8} \mathrm{~F}$ or that reason allograft (DF DBA) was chosen hypothesizing that it could enhance the effect of PRF for tissue regeneration to occur.

\section{Mechanism of Action of L- PRF :}

The L-PRF preparation process creates a gel like fibrin matrix, which incorporates platelets, leukocyte, cytokines, and circulating stem cells. PRF progressively releases cytokines over a period of time, during fibrin matrix remodelling. ${ }^{10}$ Chang et al reported that PRF activation brings about expression of phosphorylated extracellular signalregulated protein kinase (p-ERK) and osteoprotegrin (OPG) signifying its benefits for bone regeneration. Light and scanning electron microscopy showed leucocytes proliferating and interacting with osteoblasts leading to initiation in mineralization process. ${ }^{11}$ Moreover, L-PRF is capable of increasing osteoblast attachment, proliferation and simultaneously up-regulating collagen-related protein production all of which would effectively promote bone regeneration.

The matrix acts like a fibrin bandage that accelerates the healing of wound edges. It also provides a significant postoperative protection of the surgical site and seems to hasten the integration and remodeling of the grafted biomaterial. ${ }^{12}$

\section{CONCLUSION:}

According to the results obtained in the present case report, it could be concluded that the positive clinical impact of additional application of PRF with allograft in treatment of periodontal intrabony defect is based on reduction in probing pocket depth, significant radiographic defect bonefill, improved patient comfort and early wound healing process. However, long-term, multicenter randomized, controlled clinical trial will be required to know its clinical and radiographic effect over bone regeneration.

\section{REFERENCES:}

1. Giannobile WV. The potential role of growth and differentiation factors in periodontal regeneration. J Periodontol 1996; 67:545-53.

2. Froum SJ, Gomez C, Breault MR. Current concepts of periodontal regeneration. A review of the literature. $\mathrm{N} Y$ State Dent J 2002; 68:14-22.

3. Trombelli L. Which reconstructive procedures are effective for treating the periodontal intraosseous defect? Periodontol 2000 2005; 37:88-105.

4. Bartold PM, Shi S, Gronthos S. Stem cells and periodontal regeneration. Periodontol 20002006 ;40:164-72.

5. McClain PK, Schallhorn RG. Long-term assessment of combined osseous composite grafting, root conditioning, and guided tissue regeneration. Int J Periodontics Restorative Dent 1993; 13:9-27.

6. Guillemin MR, Mellonig J T, Brunsvold MA. Healing in periodontal defects treated by decalcified freeze-dried bone allografts in combination with ePTFE membranes (I). Clinical and scanning electron microscope analysis. J Clin Periodontol 1993; 20:528-36.

7. Choukroun J, Adda F, Schoeffler C, Vervelle A. The opportunity in perio-implantology: The PRF . I mplantodontie 2001; 42:55-62.

8. Lekovic V, Milinkovic I, Aleksic Z, J ankovic S, Stankovic P, Kenney EB, et al. Platel et-rich fibrin and bovine porous bone mineral vs. platelet-rich fibrin in thetreatment of intrabony periodontal defects. J Periodontal Res 2012; 47:409-17.

9. Sunitha Raja V, Munirathnam Naidu E. Platel et rich fibrin: Evolution of a second generation platelet concentration. Indian J Dent Res 2008; 19:42-6.

10. Dohan DM, Choukroun J , Diss A, et al. Platelet-rich fibrin (PRF): A second-generation platelet concentrate. Part II: Platel et-related biologic features. Oral Surg Oral Med Oral Pathol Oral Radiol Endod 2006; 101:45-50.

11. Chang I-C, Tsai C-H, Chang Y-C. Platelet-rich fibrin modulates the expression of extracellular signal-regulated protein kinase and osteoprotegerin in human osteoblasts. J Biomed Mater Res 2010; 95:327-32.

12. Kumar VR, Shubhashini N. Platelet rich fibrin: a new paradigm in periodontal regeneration. Cell Tissue Bank 2013; 14(3):453-63. 